\title{
Structural features of the O-antigen of Rickettsia typhi, the etiological agent of endemic typhus
}

\author{
M. PETUROVA ${ }^{1}$, V. VITIAZEVA², R. TOMAN ${ }^{1 *}$ \\ ${ }^{1}$ Department of Rickettsiology, Institute of Virology, Slovak Academy of Sciences, Dúbravská cesta 9, 84505 Bratislava, Slovak \\ Republic; ' ${ }^{2}$ edical Biochemistry, Gothenburg University, Box 440, 40530 Gothenburg, Sweden
}

Received February 2, 2015; accepted July 30, 2015

\begin{abstract}
Summary. - Elucidation of the O-specific polysaccharide chain of lipopolysaccharide (LPS) from Rickettsia typhi, the etiological agent of endemic typhus, is described. Structural information was established by a combination of monosaccharide and methylation analyses of the O-chain, and by mass (MS) and nuclear magnetic resonance (NMR) spectrometries of oligosaccharides arised through its hydrofluoric (HF) acid degradation. Based on the combined data from these experiments, two major polymer populations of the O-specific chain have been determined with the following structural features: $\alpha$-L-QuiNAc- $(1 \rightarrow 4)-[\alpha-D-G l c-(1 \rightarrow 3)-\alpha-L-Q u i N A c-(1 \rightarrow 4)]_{n}$ $-\alpha-D-G l c-(1 \rightarrow 4)-\alpha-D-G l c \rightarrow, \alpha-D-G l c-(1 \rightarrow 3)-\alpha-L-Q u i N A c-(1 \rightarrow 4)-[\alpha-D-G l c-(1 \rightarrow 3)-\alpha-L-Q u i N A c-(1 \rightarrow 4)]_{n}-\alpha-D-G l c \rightarrow$. The linear backbone is most probably flanked with short side chains of D-GlcNAc- $(\mathbf{1} \rightarrow \mathbf{3})-\boldsymbol{\alpha}$-L-QuiNAc- $(\mathbf{1} \rightarrow \mathbf{3})$-DGlcNAc $\rightarrow$ that are attached to it via L-QuiNAc as a branching point. It is suggested that a dimer $\boldsymbol{\alpha}$-L-QuiNAc$(\mathbf{1} \rightarrow \mathbf{3})$ - $\mathbf{a}-\mathrm{D}-\mathrm{GlcNAc}$ may represent a common epitope in the O-antigens of Proteus vulgaris OX19 and R. typhi responsible for the observed serological cross-reactivity.
\end{abstract}

Keywords: Rickettsia typhi; lipopolysaccharide; O-antigen; chemical composition; structure

\section{Introduction}

Rickettsia typhi, the causative agent of endemic (murine) typhus, is an obligate intracellular Gram-negative bacterium with a life cycle involving both vertebrate and invertebrate hosts. The classic cycle of $R$. typhi involves rats (Rattus rattus and $R$. norvegicus) and the rat flea, Xenopsylla cheopis. The

*Corresponding author. E-mail: virutoma@savba.sk; phone: +4212-59302418.

Abbreviations: COSY = correlation spectroscopy; ESI = electrospray ionization; HF acid = hydrofluoric acid; HMQC = heteronuclear multiple quantum coherence; HPLC $=$ high pressure liquid chromatography; $\mathrm{GC}=$ gas chromatography; $\mathrm{D}$-Glc = D-glucose; $\mathrm{D}$-GlcN = D-glucosamine; LPS = lipopolysaccharide; $\mathrm{MS}=$ mass spectrometry; $m / z=$ mass-to-charge ratio; L-QuiN = L-quinovosamine, 2-amino-2,6-dideoxy-L-glucose; MS/MS = tandem mass spectrometry; D-GlcNAc $=\mathrm{N}$-acetyl-D-glucosamine; $\mathrm{L}$ QuiNAc $=\mathrm{N}$-acetyl-L-quinovosamine; $\mathrm{NOE}=$ nuclear Overhauser effect; NOESY = nuclear Overhauser effect spectroscopy; OS = oligosaccharide; PS = polysaccharide flea has been considered the main vector, and the disease is transmitted by flea bites or contact with rickettsia-containing feces and tissues during or after blood feeding (Azad, 1990; Maina et al., 2012). After an incubation period of 7-14 days, the most common symptoms of endemic typhus include fever, which can last 3-7 days, headache, rash, and arthralgia (Civen and Ngo, 2008; Maina et al., 2012). The disease can be diagnosed using serological, clinical, epidemiologic and PCR methods (Maina et al., 2012). The most commonly used serological testing is quite often ambiguous due to the absence of specific and highly sensitive antigens. It is well known that R. typhi possesses at least two different types of antigens. One of them is a group-specific lipopolysaccharide (LPS) and the other is a species-specific heat-labile protein.

The chemical composition and some structural features of the LPS from R. typhi were published in the past (Amano et al., 1998) and later, we performed preliminary studies on its O-specific chain (Fodorova et al., 2005). More recently, we have established the structure of lipid A in the LPS and have shown that it displays a high degree of similarity with the classical forms of enterobacterial lipids A (Fodorova et 
al., 2011). It appears that this fact could be one of the reasons for the observed endotoxic activity of the bacterium. Further structure/function relationship studies led us to a more detailed elucidation of both chemical composition and structure of the O-specific chain of the LPS from $R$. typhi. The results are given herein.

\section{Materials and Methods}

LPS isolation and modification. R. typhi was obtained from the strain collection of the Laboratory for Diagnosis and Prevention of Rickettsial and Chlamydial Infections, Institute of Virology, Slovak Academy of Sciences, Bratislava, Slovak Republic. The bacterium was propagated in embryonated, antibiotic-free, and pathogenfree hen eggs, inactivated with $0.5 \%(\mathrm{v} / \mathrm{v})$ phenol, and purified on Renografin density gradients as described previously (Fodorova $e t$ al., 2011). LPS was isolated from purified rickettsial cells by a modified hot phenol/water procedure (Fodorova et al., 2011), purified further by ultracentrifugation $\left(105,000 \mathrm{x} \mathrm{g}, 4^{\circ} \mathrm{C}, 12 \mathrm{hr}\right)$, and the pellet was suspended in deionized water and lyophilized. The LPS was hydrolyzed with aqueous $1 \%(\mathrm{v} / \mathrm{v})$ acetic acid at $100^{\circ} \mathrm{C}$ for 1 $\mathrm{hr}$ and the hydrolyzate was kept at $-20^{\circ} \mathrm{C}$ overnight. After melting, the precipitated lipid A was removed by low-speed centrifugation (9,300 x g for $10 \mathrm{~min})$. The delipidated polysaccharide (O-antigen) was desalted with a Microsep centrifugal filter device $\left(M_{r}\right.$ cutoff 1,000; Pall Life Sciences, MI, USA). The O-antigen was treated with $48 \% \mathrm{HF}$ at $4^{\circ} \mathrm{C}$ for $48 \mathrm{hr}$, the liquid was evaporated by a stream of nitrogen, and the residual $\mathrm{O}$-antigen material was dissolved in deionized water and lyophilized. The HF-treated O-antigen was fractionated on a PL aquagel-OH 30 HPLC column $(7.5 \times 300 \mathrm{~mm}$, Agilent Technologies, USA) using deionized water as the eluant.

General and analytical methods. Analysis of the constituent sugars in the LPS was accomplished by the alditol acetate method (Sawardeker et al., 1965). The LPS was hydrolyzed with $2 \mathrm{~mol} / \mathrm{l}$ trifluoroacetic acid at $120^{\circ} \mathrm{C}$ for $3 \mathrm{hr}$ and this was followed by reduction with sodium borohydride and subsequent acetylation with the acetic anhydride-pyridine mixture. Alditol acetate derivatives were analyzed as previously described (Fodorova et al., 2011). Methylation analyses of the HF-treated $\mathrm{O}$-antigen and the oligosaccharide fraction (OS) were carried out according to Ciucanu and Kerek (1984) and with characterization of permethylated alditol acetate derivatives by GC-MS. For HPLC-ESI-MS analyses, the OS was methylated with methyl iodide in the presence of butyllithium (Kvernheim, 1987).

HPLC-ESI-MS. The permethylated OS dissolved in $1 \mathrm{mmol} / \mathrm{l}$ sodium acetate in methanol-water $(7: 3, \mathrm{v} / \mathrm{v})$ was analyzed with an HPLC Waters 2690 (Waters, Milford, USA) coupled to a Finnigan LCQ-trap mass spectrometer (Finnigan-MAT, San Jose, CA, USA). The sample was separated on a LUNA C18(2) column (1 x $150 \mathrm{~mm}$, Phenomenex) using a gradient of solutions A: $1 \mathrm{mmol} / \mathrm{l}$ sodium acetate $-1 \%(\mathrm{v} / \mathrm{v})$ acetic acid in methanol and B: $1 \mathrm{mmol} / \mathrm{l}$ sodium acetate $-1 \%(\mathrm{v} / \mathrm{v})$ acetic acid in water. The gradient program was
$50 \%$ A increased to $100 \%$ in $50 \mathrm{~min}$, and $100 \%$ A for $20 \mathrm{~min}$ at a flow rate of $0.1 \mathrm{ml} \cdot \mathrm{min}^{-1}$. The experiments were run in a positive ion mode.

NMR. NMR spectra of OS were recorded on the Varian 600$\mathrm{MHz}$ spectrometer, with standard software. All NMR experiments were performed at $70^{\circ} \mathrm{C}$, with acetone as an internal reference at $\delta$ $2.225 \mathrm{ppm}$ for ${ }^{1} \mathrm{H}$ spectra and $31.45 \mathrm{ppm}$ for ${ }^{13} \mathrm{C}$ spectra. Standard homo- and heteronuclear correlated 2D pulse sequences from Varian, correlation spectroscopy (COSY), nuclear Overhauser effect spectroscopy (NOESY), and heteronuclear multiple quantum coherence (HMQC) were used for general assignments.

\section{Results and Discussion}

\section{Isolation and characterization of LPS from R. typhi}

LPS was isolated from $R$. typhi cells by a modified hot phenol/water extraction procedure. It was found in phenol layer, and after dialysis and lyophilization, purified further by ultracentrifugation. The sugar analysis of the LPS revealed the presence of glucose (Glc), quinovosamine (QuiN, 2-amino-2,6-dideoxy-glucose), and glucosamine (GlcN) in an approximate molar ratio of 1.3:1.0:0.3, respectively. Glc and GlcN were shown to have a D-configuration, in contrast with QuiN that indicated an L-configuration (Gerwig et al., 1978). Only traces of L-glycero-D-manno-heptose were detected. Analysis of fatty acids performed in the previous work (Fodorova et al., 2011) revealed the presence of 3-hydroxytetradecanoic, 3-hydroxyhexadecanoic, hexadecanoic, and octadecanoic acids in a molar ratio 2.2:2.1:1.3:1.0, respectively.

Partial depolymerization of the O-antigen and methylation analyses of selected degradation products

The O-antigen isolated from the parent LPS by treatment with mild acid was treated with $\mathrm{HF}$ and separated on a gel column using HPLC. Both HF-treated O-antigen and the major oligosaccharide (OS) fraction eluted from the column were subjected to methylation analyses. These showed mainly the presence of 4-substituted D-Glc and 3-substituted Nacetyl-L-quinovosamine (L-QuiNAc), together with lesser amounts of terminal D-Glc, L-QuiNAc and N-acetyl-Dglucosamine (D-GlcNAc), and 3-substituted D-GlcNAc in the analyzed samples.

\section{HPLC-ESI-MS analysis of the methylated OS}

The methylated OS was analyzed further by HPLC-ESI-MS with the aim to investigate both composition and sequence of the present glycoforms. In the MS spectrum (Fig. 1), twelve 


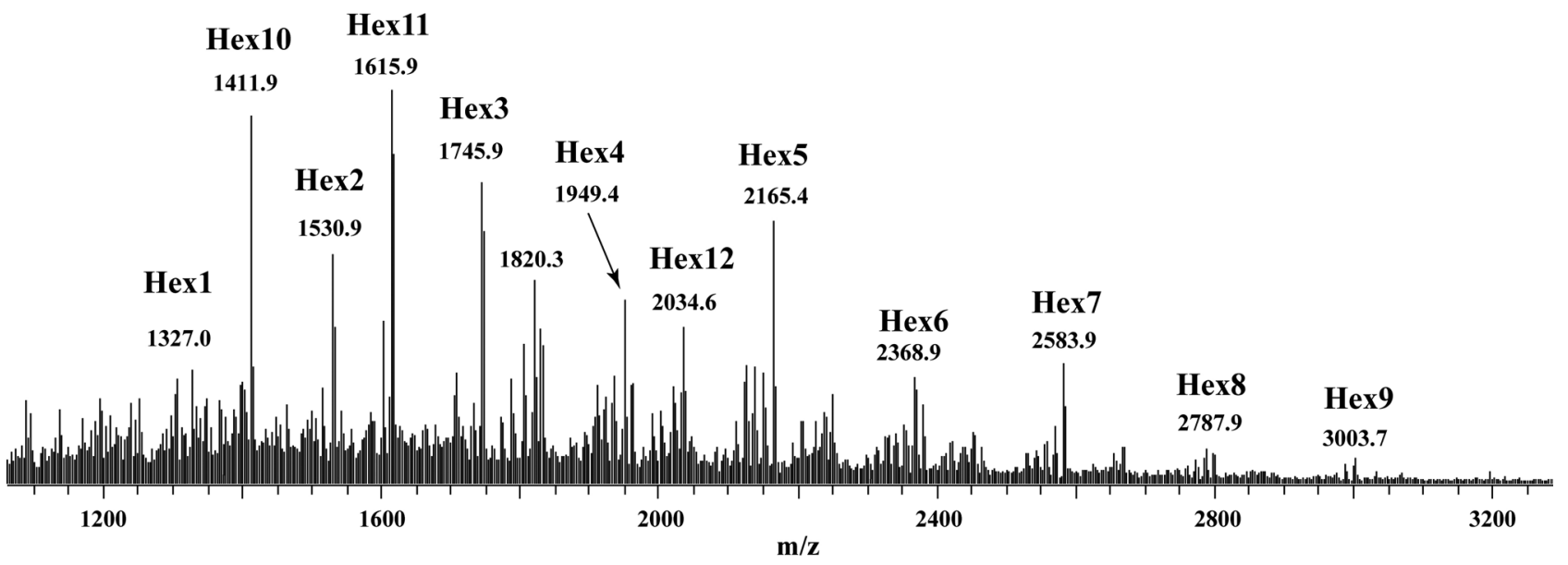

Fig. 1

The positive ion mode ESI-MS spectrum of the permethylated OS fraction from HF-treated PS of R. typhi. For Hex1-Hex12 (see Table 1)

$[\mathrm{M}+\mathrm{Na}]^{+}$ions were observed at $\mathrm{m} / z 1327.0,1530.9,1745.9$, 1949.4, 2165.4, 2368.9, 2583.9, 2787.9 and 3003.7 (Hex1Hex9), and at $m / z 1411.9,1615.9$ and 2034.6 (Hex10-Hex12). The proposed compositions and sequences of the glycoforms are given in Table 1 . In the analysis, the Hex3-Hex12 ions were subjected to $\mathrm{MS}^{2}$ and the ions at $m / z 1929$ and 2151 to $\mathrm{MS}^{3}$ experiments. The resulting fragmentations depicted in the corresponding MS spectra (Supplements 1-10) have unambiguously confirmed compositions and sequences of the methylated oligomers listed in Table 1.
The combined results of methylation-linkage analysis and ESI-MS measurements indicated that the O-chain of the LPS from $R$. typhi was mainly composed of the alternating 4-substituted D-Glc and 3-substituted L-QuiNAc residues. Terminal L-QuiNAc appeared to be more frequent at the non-reducing end of the O-chain than D-Glc. The 4-substituted D-Glc prevailed at the reducing end. In contrast with other glycoforms found, the oligomers Hex10Hex12 displayed a terminal sequence containing D-GlcNAc that alternated with L-QuiNAc. The methylation analysis

Table 1. ESI-MS analysis of the methylated OS from HF-treated PS of $R$. $t y p h i$ and proposed compositions and sequences of the glycoforms Hex1-Hex 12

\begin{tabular}{|c|c|c|c|c|}
\hline \multirow{2}{*}{$\begin{array}{l}\text { Ions } \\
{[\mathrm{M}+\mathrm{Na}]^{+}}\end{array}$} & \multicolumn{2}{|c|}{ Molecular mass } & \multirow{2}{*}{ Proposed composition } & \multirow{2}{*}{ Glycoform } \\
\hline & Observed & Calculated $^{\mathrm{a}}$ & & \\
\hline 1327.0 & 1304.0 & 1304.5 & btQuiNAc-[Glc-QuiNAc] $]_{2}-$ Glc-OCH$_{3}$ & Hex1 \\
\hline 1530.9 & 1507.9 & $\begin{array}{l}1508.7 \\
1508.7\end{array}$ & $\begin{array}{l}\text { tQuiNAc-[Glc-QuiNAc] }- \text {-Glc-Glc-OCH } \\
\text { tGlc-QuiNAc-[Glc-QuiNAc] }- \text {-Glc-OCH }\end{array}$ & Hex2 \\
\hline 1745.9 & 1722.9 & 1723.9 & tQuiNAc-[Glc-QuiNAc] $]_{3}-\mathrm{Glc}_{\mathrm{OCCH}}$ & Hex3 \\
\hline 1949.4 & 1926.4 & $\begin{array}{l}1928.2 \\
1928.2\end{array}$ & $\begin{array}{l}\text { tQuiNAc-[Glc-QuiNAc] }- \text {-Glc-Glc-OCH } \\
\text { tGlc-QuiNAc-[Glc-QuiNAc] }{ }_{3}-\mathrm{Glc}-\mathrm{OCH}_{3}\end{array}$ & Hex4 \\
\hline 2165.4 & 2142.4 & 2143.4 & tQuiNAc-[Glc-QuiNAc] $]_{4}-\mathrm{Glc}_{-} \mathrm{OCH}_{3}$ & Hex 5 \\
\hline 2368.9 & 2345.9 & $\begin{array}{l}2347.6 \\
2347.6\end{array}$ & $\begin{array}{l}\text { tQuiNAc-[Glc-QuiNAc] }- \text {-Glc-Glc-OCH } \\
\text { tGlc-QuiNAc-[Glc-QuiNAc] }]_{4}-\text { Glc-OCH }\end{array}$ & Hex6 \\
\hline 2583.9 & 2560.9 & 2562.9 & tQuiNAc-[Glc-QuiNAc] $]_{5}-\mathrm{Glc}_{-} \mathrm{OCH}_{3}$ & Hex7 \\
\hline 2787.9 & 2764.9 & $\begin{array}{l}2767.1 \\
2767.1\end{array}$ & $\begin{array}{l}\text { tQuiNAc-[Glc-QuiNAc] }- \text {-Glc-Glc-OCH } \\
\text { tGlc-QuiNAc-[Glc-QuiNAc] }]_{5}-\mathrm{Glc}_{3} \mathrm{OCH}_{3}\end{array}$ & Hex8 \\
\hline 3003.7 & 2980.7 & 2982.3 & tQuiNAc-[Glc-QuiNAc] $]_{6}-\mathrm{Glc}-\mathrm{OCH}_{3}$ & Hex9 \\
\hline 1411.9 & 1388.9 & 1386.6 & tGlcNAc-QuiNAc-GlcNAc-QuiNAc-QuiNAc-Glc-OCH ${ }_{3}$ & Hex10 \\
\hline 1615.9 & 1592.9 & 1590.8 & tGlcNAc-QuiNAc-GlcNAc-QuiNAc-Glc-QuiNAc-Glc-OCH ${ }_{3}$ & Hex11 \\
\hline 2034.6 & 2011.6 & 2010.3 & tGlcNAc-QuiNAc-GlcNAc-[QuiNAc-Glc] ${ }_{2}$-QuiNAc-Glc-OCH ${ }_{3}$ & Hex12 \\
\hline
\end{tabular}

${ }^{a}$ Molecular masses were calculated using the following average mass units: Glc, 162.1; QuiNAc, 187.2; GlcNAc, 203.2; Me, 15.0; Na, 23.0; $\mathrm{H}_{2} \mathrm{O}, 18.0$. ${ }^{b} t$, terminal. For the corresponding MS spectra of Hex3-Hex12 and relevant fragmentations, see supplements 1-10. 


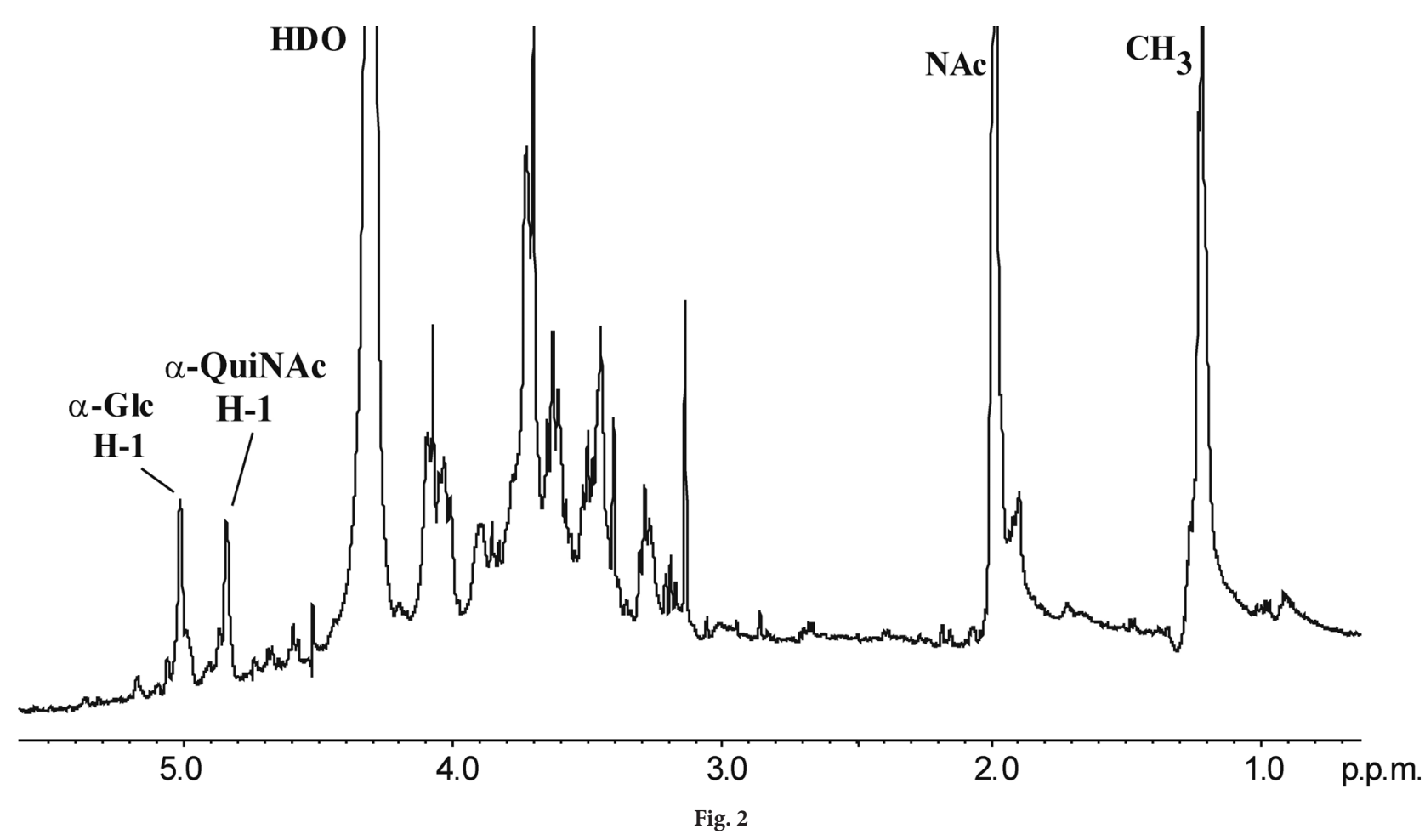

${ }^{1} \mathrm{H}$-NMR spectrum of OS from $R$. typhi with anomeric protons assigned for $\alpha$-QuiNAc and $\alpha$-Glc

indicated that the internal D-GlcNAc residues were linked by $1 \rightarrow 3$ bonds.

\section{NMR analysis of $O S$}

The ${ }^{1} \mathrm{H}$ spectrum of the OS sample showed signals for the anomeric protons of L-QuiNAc and D-Glc at $\delta 4.84$ and 5.02 , respectively (Fig. 2). The spectrum showed also a group of signals corresponding to the ring protons in the region of $\delta$ 3.1-4.1. The signals for methyl $\left(\mathrm{CH}_{3}\right)$ groups of the 6 -deoxy sugar and $\mathrm{N}$-acetyl groups were recorded at $\delta 1.22$ and 2.00, respectively. In the COSY spectrum (not shown), two $\mathrm{H}-1 / \mathrm{H}-2$ cross-peaks corresponding to $\alpha$-L-QuiNAc and $\alpha$-D-Glc were identified at $\delta_{\mathrm{H}} 4.09$ and 3.45, respectively. The proton and carbon chemical shifts of both sugar residues were mostly assigned based on HMQC experiments. Part of the HMQC spectrum showing most of the assigned signals is given in Fig. 3. The signals for $\mathrm{N}$-acetyl and $\mathrm{CH}_{3}$ groups of L-QuiNAc were found at $\delta_{\mathrm{H} / \mathrm{C}} 2.00 / 22.0$ and $1.22 / 17.5$, respectively.

The ${ }^{1} \mathrm{H}$ and ${ }^{13} \mathrm{C}$ NMR resonances were assigned using COSY, HMQC and NOESY experiments and are summarized in Table 2 . Based on relatively small $\mathrm{J}_{1,2}$ values and ${ }^{13} \mathrm{C}$ anomeric shifts from the ${ }^{1} \mathrm{H}-{ }^{13} \mathrm{C}$ HMQC experiment, both sugars were identified as having the $a$-anomeric configuration. This was also confirmed by intra-residue NOE connectivities between the protons $\mathrm{H}-1$ and $\mathrm{H}-2$. The absence of carbon signals in the region of $\delta_{C} 80-88$, which are characteristic for furanosides, indicated that D-Glc and L-QuiNAc are in the pyranose form. The presence of amino

Table 2. ${ }^{1} \mathrm{H}$ - and ${ }^{13} \mathrm{C}$-NMR chemical shifts for OS separated on a gel column of HF-treated PS from $R$. $t y p h i$

\begin{tabular}{|c|c|c|c|c|c|c|c|c|}
\hline Sugar unit & Linkage & $\mathrm{H} / \mathrm{C} 1$ & $\mathrm{H} / \mathrm{C} 2$ & $\mathrm{H} / \mathrm{C} 3$ & $\mathrm{H} / \mathrm{C} 4$ & $\mathrm{H} / \mathrm{C} 5$ & $\mathrm{H} / \mathrm{C} 6$ & \\
\hline \multirow[t]{2}{*}{ QuiNAc } & $\rightarrow 3)$-a-QuiNAcp-(1 $\rightarrow$ & 4.84 & 4.09 & 3.72 & 3.27 & 4.07 & 1.22 & \\
\hline & & 98.3 & 54.6 & 79.4 & 75.0 & 69.3 & 17.5 & \\
\hline \multirow[t]{2}{*}{ Glc } & $\rightarrow 4)-\alpha-$ Glc $p-(1 \rightarrow$ & 5.01 & 3.45 & 3.45 & 3.50 & 3.45 & 3.60 & 3.71 \\
\hline & & 100.1 & 72.5 & 72.5 & 77.8 & 72.5 & 60.9 & \\
\hline
\end{tabular}

Data were recorded in $\mathrm{D}_{2} \mathrm{O}$ at $70^{\circ} \mathrm{C}$; referenced to internal acetone at 2.225/31.45 ppm. 


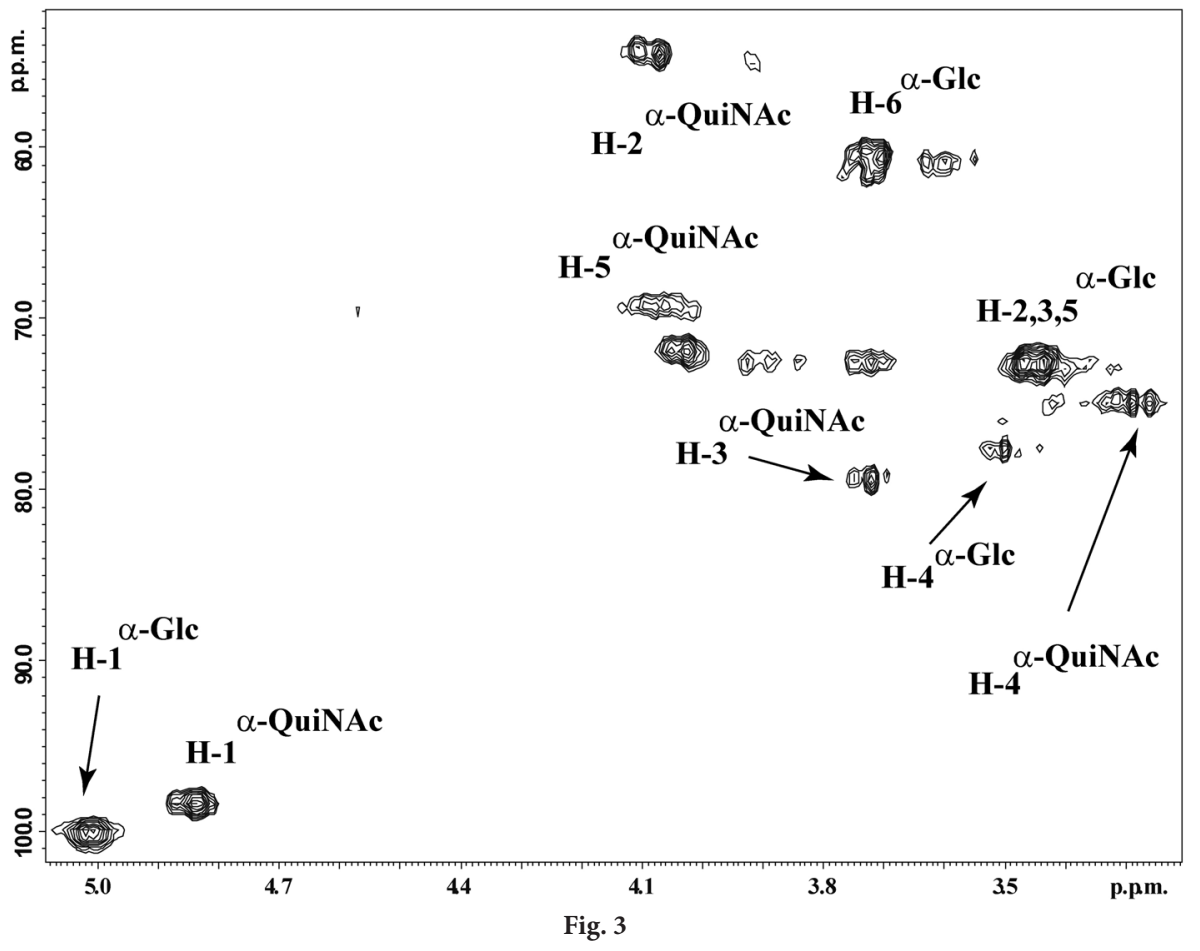

Part of the ${ }^{1} \mathrm{H}-{ }^{13} \mathrm{C}$ - HMQC spectrum of $\mathrm{OS}$ from $R$. typhi with the assigned signals

sugar was also confirmed by correlation of the proton at the nitrogen-linked C-2 to the corresponding carbon at $\delta_{\mathrm{H} 2} / \delta_{\mathrm{C} 2}$ 4.09/54.6. The occurrence of inter-residue NOESY connectivities between the proton pairs $\mathrm{H}-1$ of $\alpha$-L-QuiNAc and $\mathrm{H}-4$ of $\alpha-\mathrm{D}-\mathrm{Glc}$ indicated that $\alpha-\mathrm{D}-\mathrm{Glc}$ was substituted at the O-4 position by $\alpha$-L-QuiNAc, which was substituted by $a-D-G l c$ residue at the $\mathrm{O}-3$ position as found from the NOE connectivities between the proton pairs $\mathrm{H}-1$ of $\alpha-\mathrm{D}-\mathrm{Glc}$ and H-3 of $\alpha$-L-QuiNAc .

Based on the combined data from the experiments mentioned above, two major polymer populations of the O-specific chain have the following structural features: $\boldsymbol{\alpha}$-L-QuiNAc$(1 \rightarrow 4)$-[ $\alpha-D-G l c-(1 \rightarrow 3)$ - $\alpha-L-Q u i N A c-(1 \rightarrow 4)]_{n}-\alpha-D-G l c-$ $(1 \rightarrow 4)-\alpha-D-G l c \rightarrow, \alpha-D-G l c-(1 \rightarrow 3)-\alpha-L-Q u i N A c-(1 \rightarrow 4)-[\alpha-D-$ Glc- $(1 \rightarrow 3)-\alpha-L-Q u i N A c-(1 \rightarrow 4)]_{n}-\boldsymbol{\alpha}-D-G l c \rightarrow$. The oligomers Hex10-Hex12 indicate that the linear backbone of the $\mathrm{O}$-antigen is most probably flanked with short side chains of D-GlcNAc- $(1 \rightarrow 3)$ - $\alpha$-L-QuiNAc- $(1 \rightarrow 3)$-D-GlcNAc $\rightarrow$ that are attached to it via L-QuiNAc as a branching point. However, the mode of their attachment to L-QuiNAc and the anomeric configuration of the D-GlcNAc residues have remained unknown thus far.

Serodiagnosis of rickettsiosis employed Proteus group OX strains as antigens, which gave characteristic agglutination patterns with sera from patients infected with various rickettsial agents (Amano et al., 1995, 1998). Thus, sera from patients with typhus and Rocky Mountain spotted fever reacted with $P$. vulgaris OX19, whereas $P$. vulgaris OX2 cells were agglutinated by sera from patients with spotted fever (except for Rocky Mountain spotted fever). Chemical structures of O-antigens of both Proteus bacteria were determined in the past (Ziolkowski et al. 1997). The PS moiety of strain OX2 LPS contained D-Glc, D-GlcNAc, and L-QuiNAc, whereas the PS moiety of strain OX19 LPS contained D-galactose, D-GlcNAc, N-acetyl-D-galactosamine, and L-QuiNAc. Both $\mathrm{O}$-antigens also differed in the structural arrangement of their repeating units. This study shows that the $\mathrm{O}$-antigen of $R$. typhi has also a distinct chemical composition and structural features from those reported for the OX2 and OX19 antigens. The observed serological cross-reactivity between $P$. vulgaris OX19 and $R$. typhi indicates that both bacteria utilize the same epitope or similar epitopes in their O-antigens. In their work, Zych et al., 2005, suggested a dimer $\alpha$-L-QuiNAc- $(1 \rightarrow 3)-\alpha-D$ GlcNAc as a common serologically active epitope in the O-antigens of $P$. vulgaris OX19, P. penneri S29 and 26 LPSs. This sugar sequence is also found in the short side chains of the O-PS backbone of $R$. typhi LPS and could represent a binding site for human anti-typhus antibodies. In the $\mathrm{O}$-antigen of $P$. vulgaris OX2, the epitope suggested to be responsible for serological reactions represents a disaccharide $\alpha$-L-QuiNAc- $(1 \rightarrow 3)-\beta-D-G l c N A c 6 A c$ that apparently does not bind with human anti-rickettsial antibodies. This data further support a known fact that subtle changes in 
the structural features of investigated epitopes may influence greatly their serological reactivity.

Supplementary information is available in the online version of the paper.

Acknowledgements. This work was supported in part by the grant 2/0026/12 from the Scientific Grant Agency of Ministry of Education of Slovak Republic and the Slovak Academy of Sciences. The authors wish to thank Prof. Elke Schweda from the Department of Physics, Chemistry and Biology of the Linköping University for her interest and support of this work.

\section{References}

Amano K, Kyohno K, Aoki S, Suto T (1995): Serological studies of the antigenic similarity between typhus group rickettsiae and Weil-Felix test antigens. Microbiol. Immunol. 39, 63-65. http://dx.doi.org/10.1111/j.1348-0421.1995.tb02169.x

Amano K, Williams JC, Dasch GA (1998): Structural properties of lipopolysaccharides from Rickettsia typhi and Rickettsia prowazekii and their chemical similarity to the lipopolysaccharide from Proteus vulgaris OX19 used in the Weil-Felix test. Infect. Immun. 66, 923-926.

Azad AF (1990): Epidemiology of murine typhus. Annu. Rev. Entomol. 35, 553-559. http://dx.doi.org/10.1146/annurev. en.35.010190.003005

Ciucanu I, Kerek F (1984): A simple and rapid method for the permethylation of carbohydrates. Carbohydr. Res. 131, 209-217. http://dx.doi.org/10.1016/0008-6215(84) $85242-8$

Civen R, Ngo V (2008): Murine Typhus: An unrecognized suburban vectorborne disease. Clin. Infect. Dis. 46, 913-918. http:// dx.doi.org/10.1086/527443
Fodorova M, Vadovic P, Skultety L, Slaba K, Toman R (2005): Structural features of lipopolysaccharide from Rickettsia typhi, the causative agent of endemic typhus. Ann. N.Y. Acad. Sci. 1063, 259-260. http://dx.doi.org/10.1196/annals.1355.041

Fodorova M, Vadovic P, Toman R (2011): Structural features of lipid A of Rickettsia typhi. Acta Virol. 55, 31-44. http:// dx.doi.org/10.4149/av $201101 \quad 31$

Gerwig GJ, Kamerling JP, Vliegenthart JF (1978): Determination of the D and L configuration of neutral monosaccharides by high-resolution capillary g.l.c. Carbohydr. Res. 62, 349-357. http://dx.doi.org/10.1016/S0008-6215(00)80881-2

Kvernheim AL (1987): Methylation analysis of polysaccharides with butyllithium in dimethyl sulfoxide. Acta Chem. Scand. B41, 150-152. http://dx.doi.org/10.3891/acta. chem.scand.41b-0150

Maina AN, Speck S, Spitalska E, Toman R, Dobler G, Cutler SJ (2012): Rickettsia species: Rickettsioses. In Elschner MC, Cutler SJ, Weidmann M, Butaye P (Eds): BSL3 and BSL4 Agents. Epidemiology, Microbiology, and Practical Guidelines. Wiley-VCH Verlag GmbH \& Co. KGaA, pp. 123-148. http://dx.doi.org/10.1002/9783527645114. ch8

Sawardeker JS, Sloneker JH, Jeanes A (1965): Quantitative determination of monosaccharides as their alditol acetates by gas liquid chromatography. Anal. Chem. 37, 1602-1603. http://dx.doi.org/10.1021/ac60231a048

Ziolkowski A, Shashkov AS, Swierzko AS, Senchenkova SN, Toukach FV, Cedzynski M, Amano KI, Kaca W, Knirel YA (1997): Structures of the O-antigens of Proteus bacilli belonging to OX group (serogroups O1-O3) used in Weil-Felix test. FEBS Lett. 411, 221-224. http://dx.doi. org/10.1016/S0014-5793(97)00694-7

Zych K, Siwinska M, Sidorczyk Z (2005): Serological classification and epitope specificity of Proteus penneri S29 lipopolysaccharide. Arch. Immunol. Ther. Exp. 53, 540-545. 


\title{
Supplementary information
}

\section{Structural features of the O-antigen of Rickettsia typhi, the etiological agent of endemic typhus}

\author{
M. PETUROVA ${ }^{1}$, V. VITIAZEVA ${ }^{2}$, R. TOMAN ${ }^{1 *}$
}

${ }^{1}$ Department of Rickettsiology, Institute of Virology, Slovak Academy of Sciences, Dúbravská cesta 9, 84505 Bratislava, Slovak Republic; ${ }^{2}$ Medical Biochemistry, Gothenburg University, Box 440, 40530 Gothenburg, Sweden

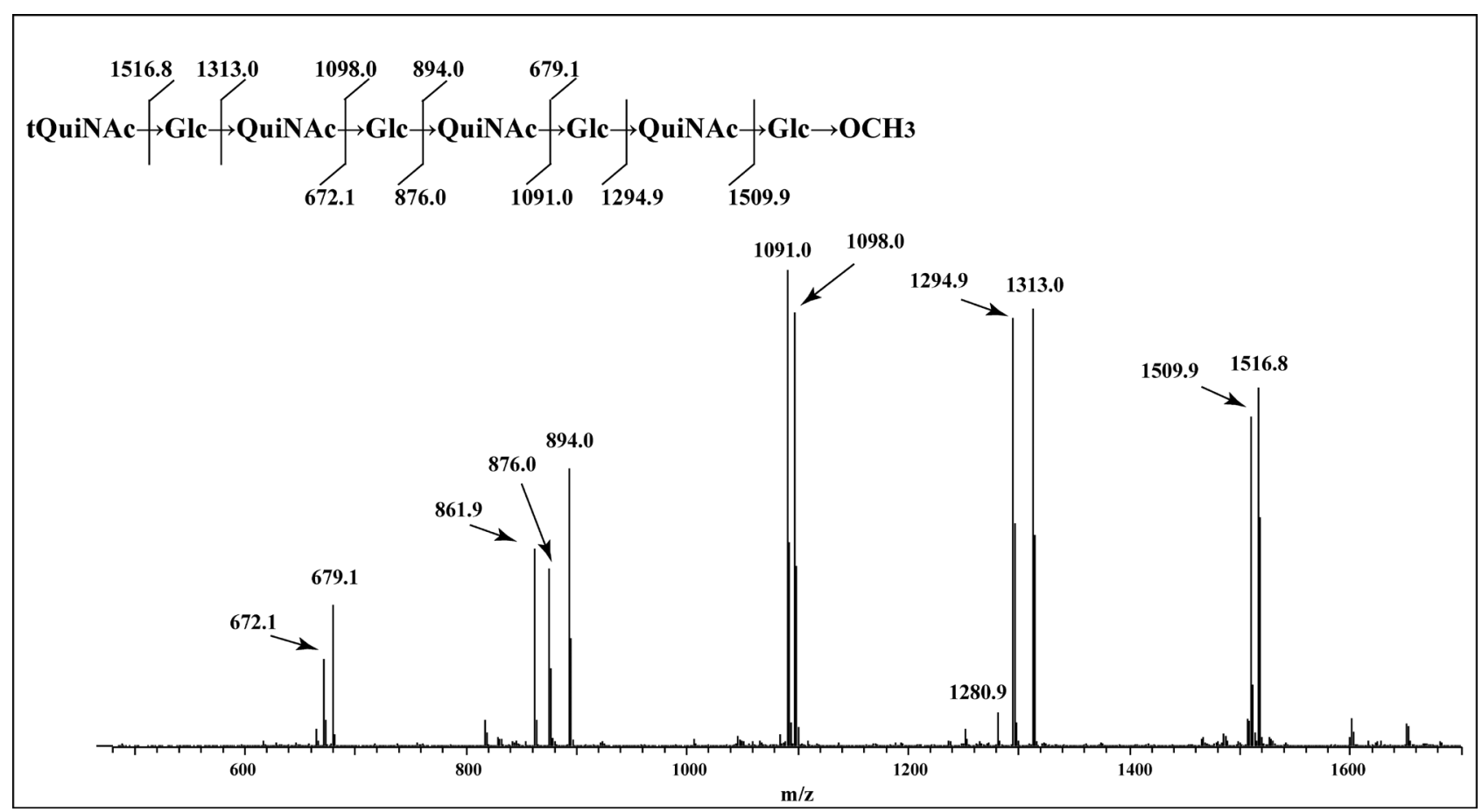

Supplement 1

ESI-MS $^{2}$ spectrum and the fragmentation scheme of the glycoform Hex3 at $m / z$ 1746; $t$, terminal 


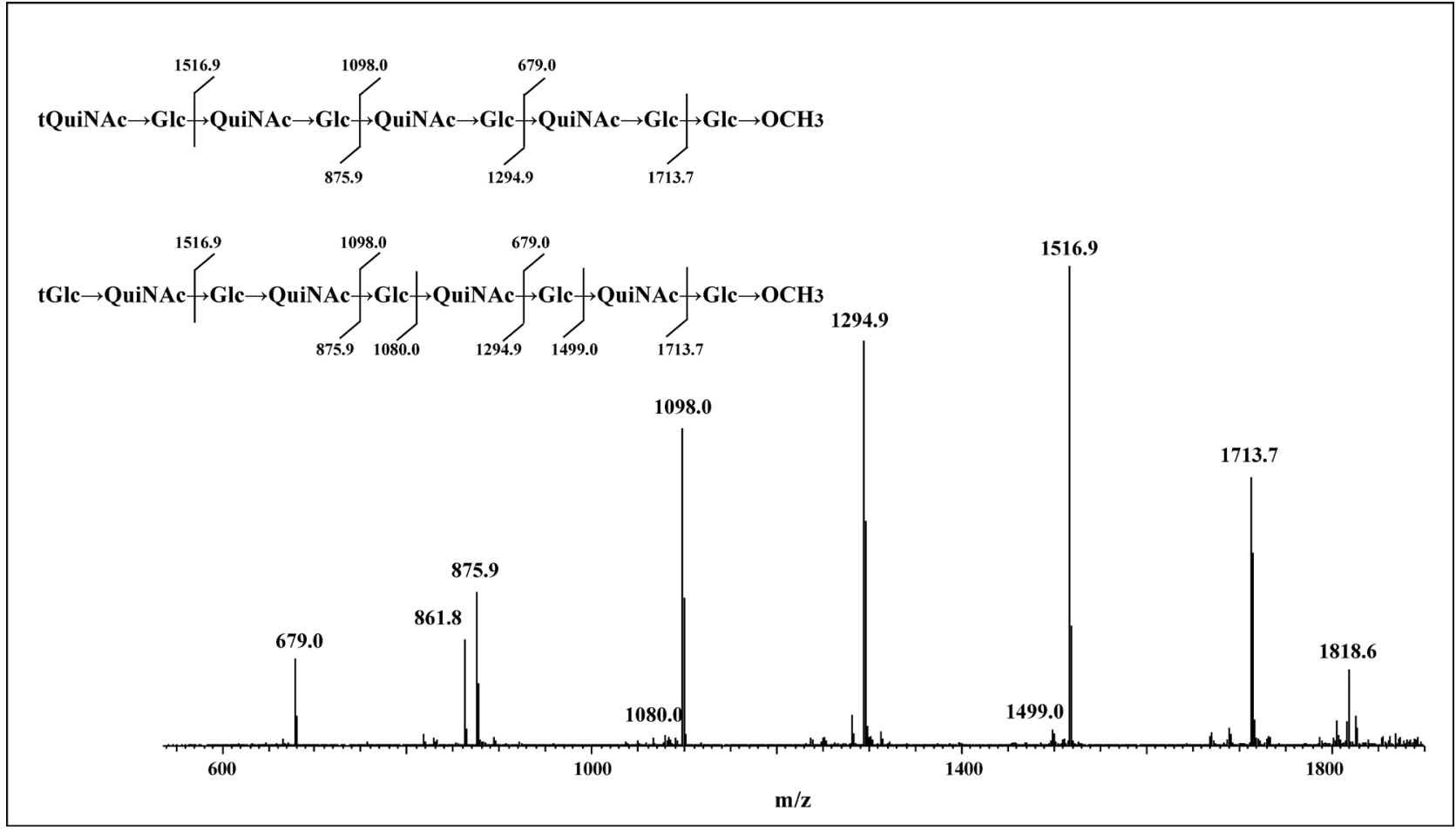

Supplement 2

ESI-MS ${ }^{2}$ spectrum and the fragmentation schemes of the glycoform Hex4 at $m / z$ 1950; $t$, terminal

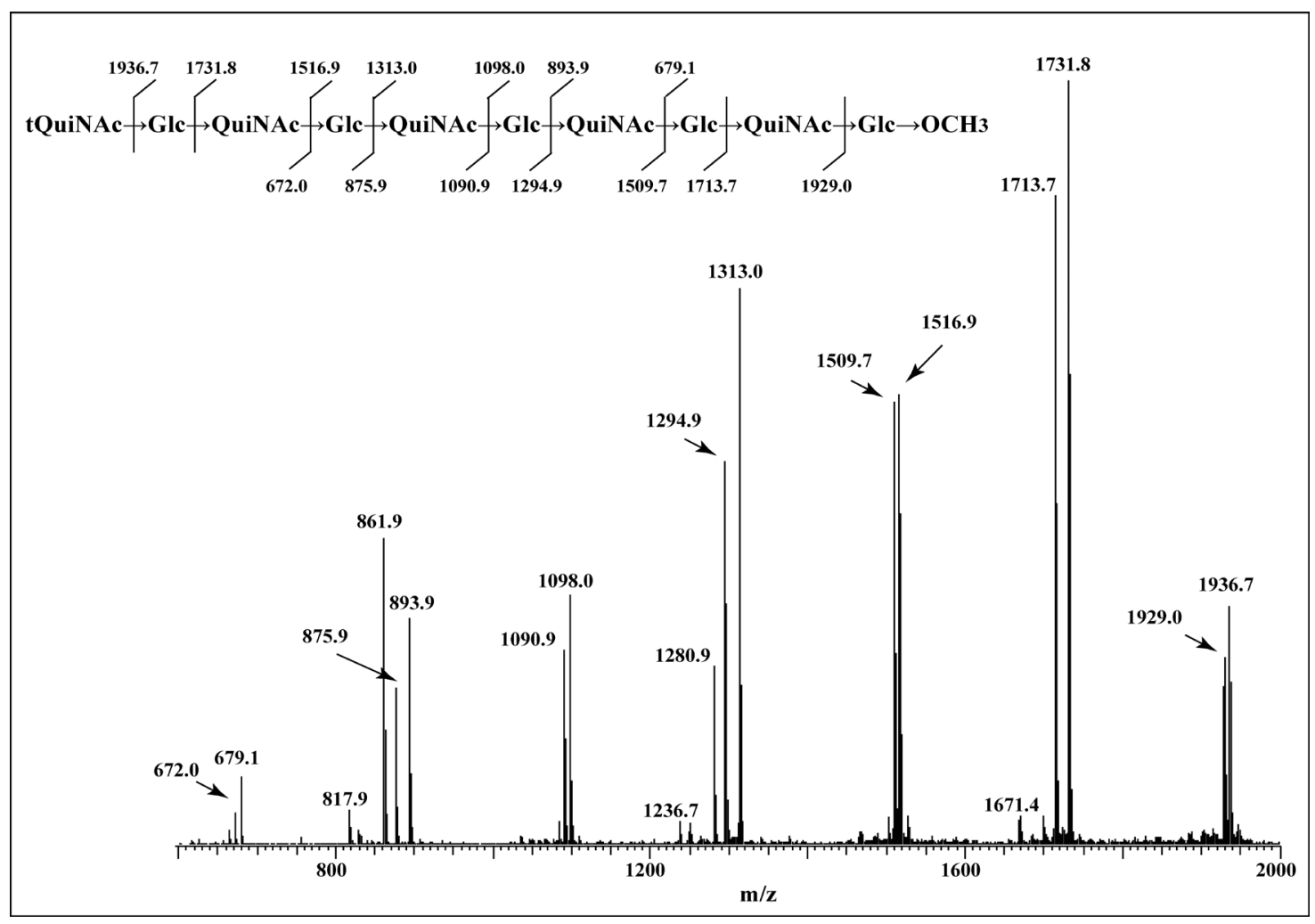

Supplement 3

ESI-MS ${ }^{2}$ spectrum and the fragmentation scheme of the glycoform Hex 5 at $m / z 2165$; t terminal 


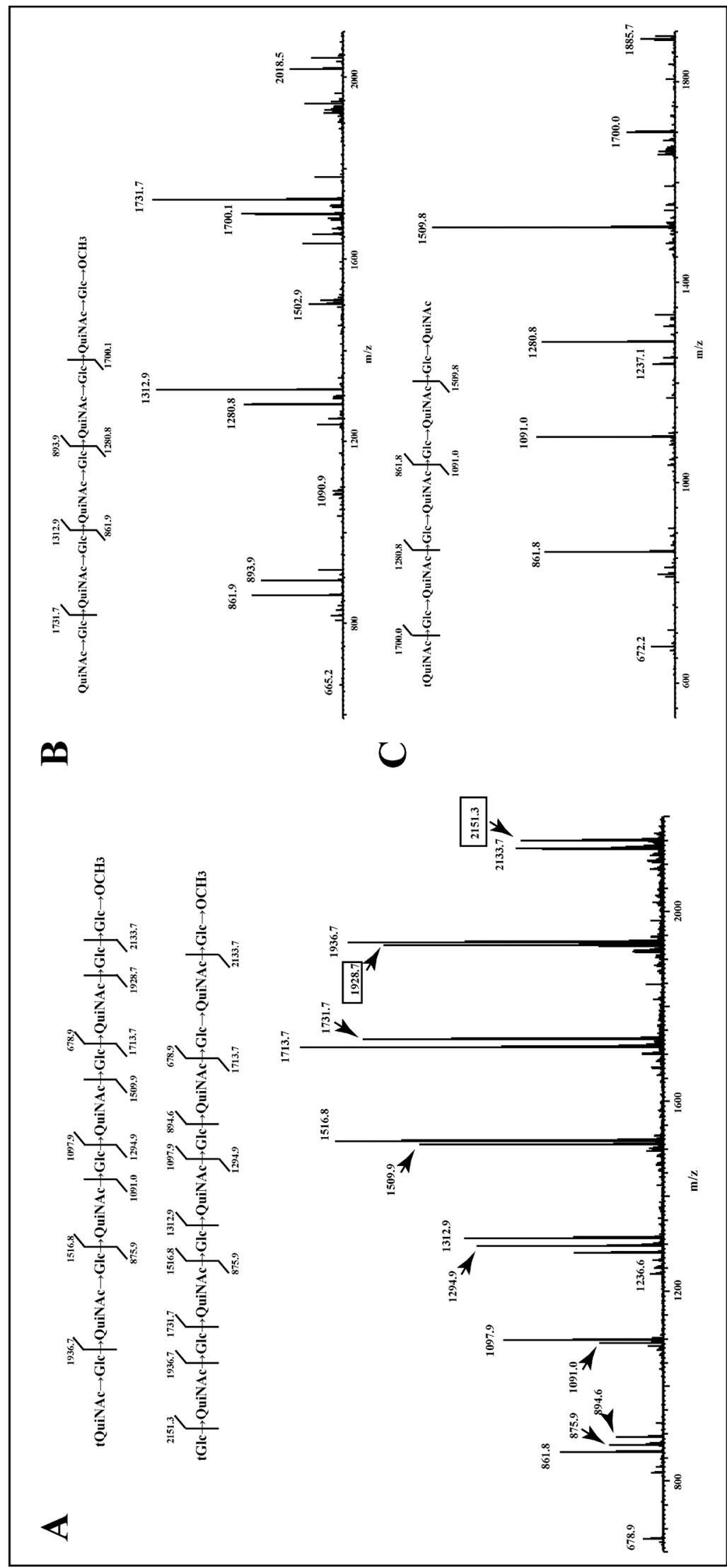

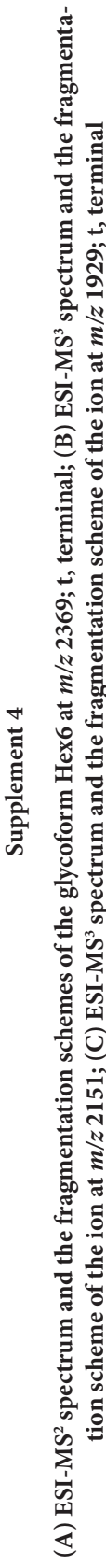




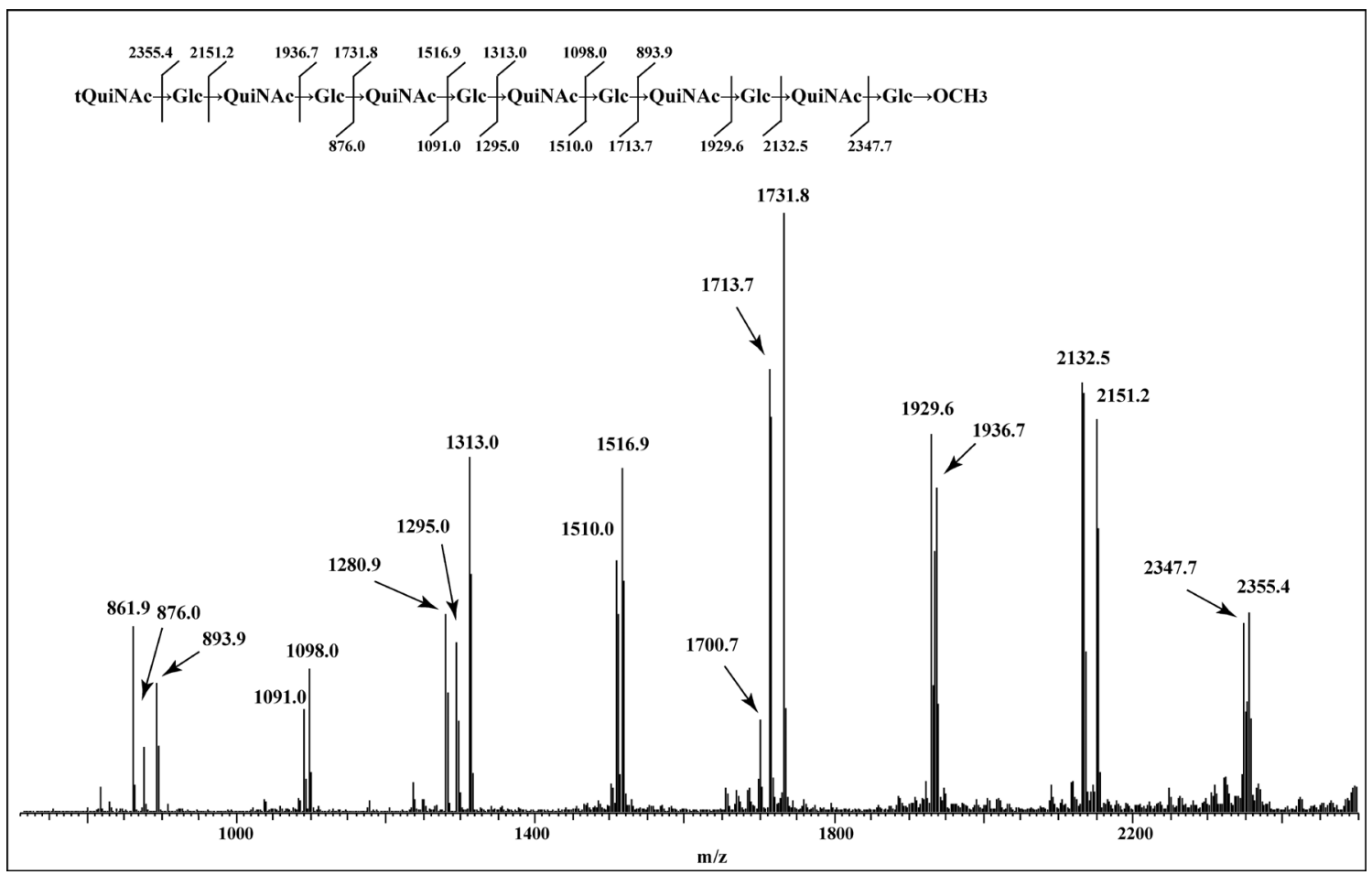

Supplement 5

ESI-MS $^{2}$ spectrum and the fragmentation scheme of the glycoform Hex7 at $m / z 2584 ; t$, terminal

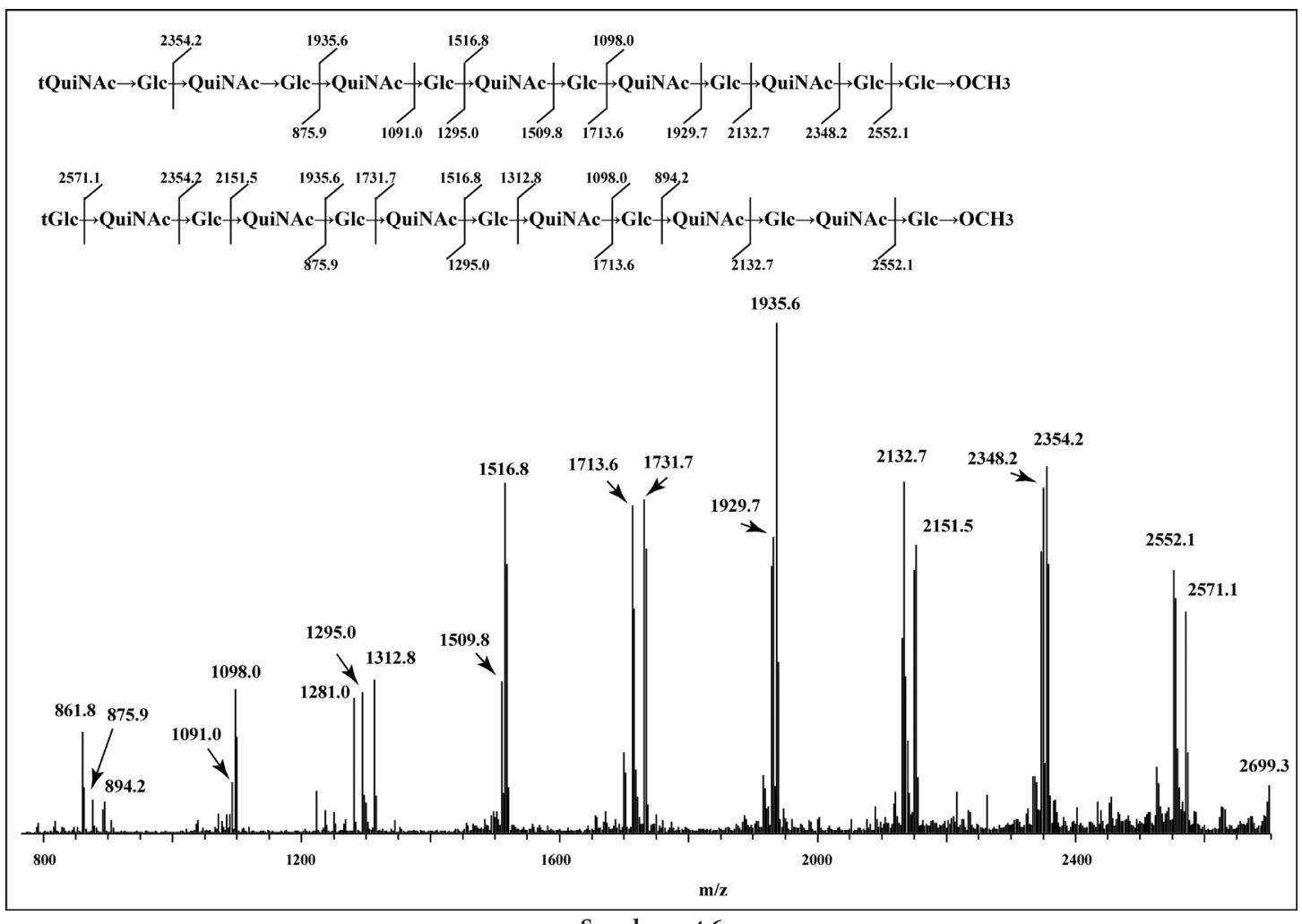

Supplement 6

ESI-MS ${ }^{2}$ spectrum and the fragmentation schemes of the glycoform Hex8 at $m / z 2788$; $t$, terminal 


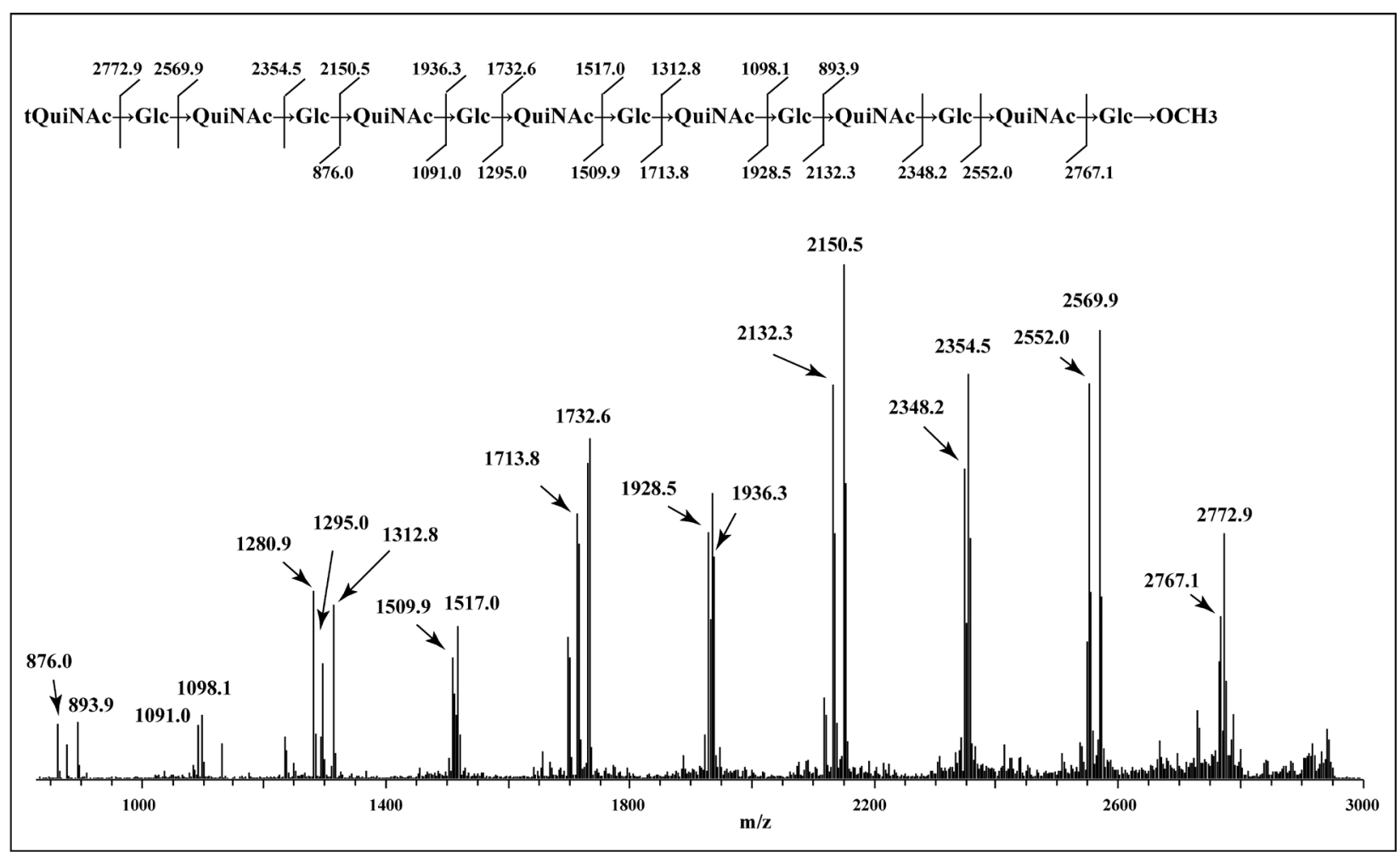

Supplement 7

ESI-MS ${ }^{2}$ spectrum and the fragmentation scheme of the glycoform Hex9 at $m / z 3003$; t, terminal

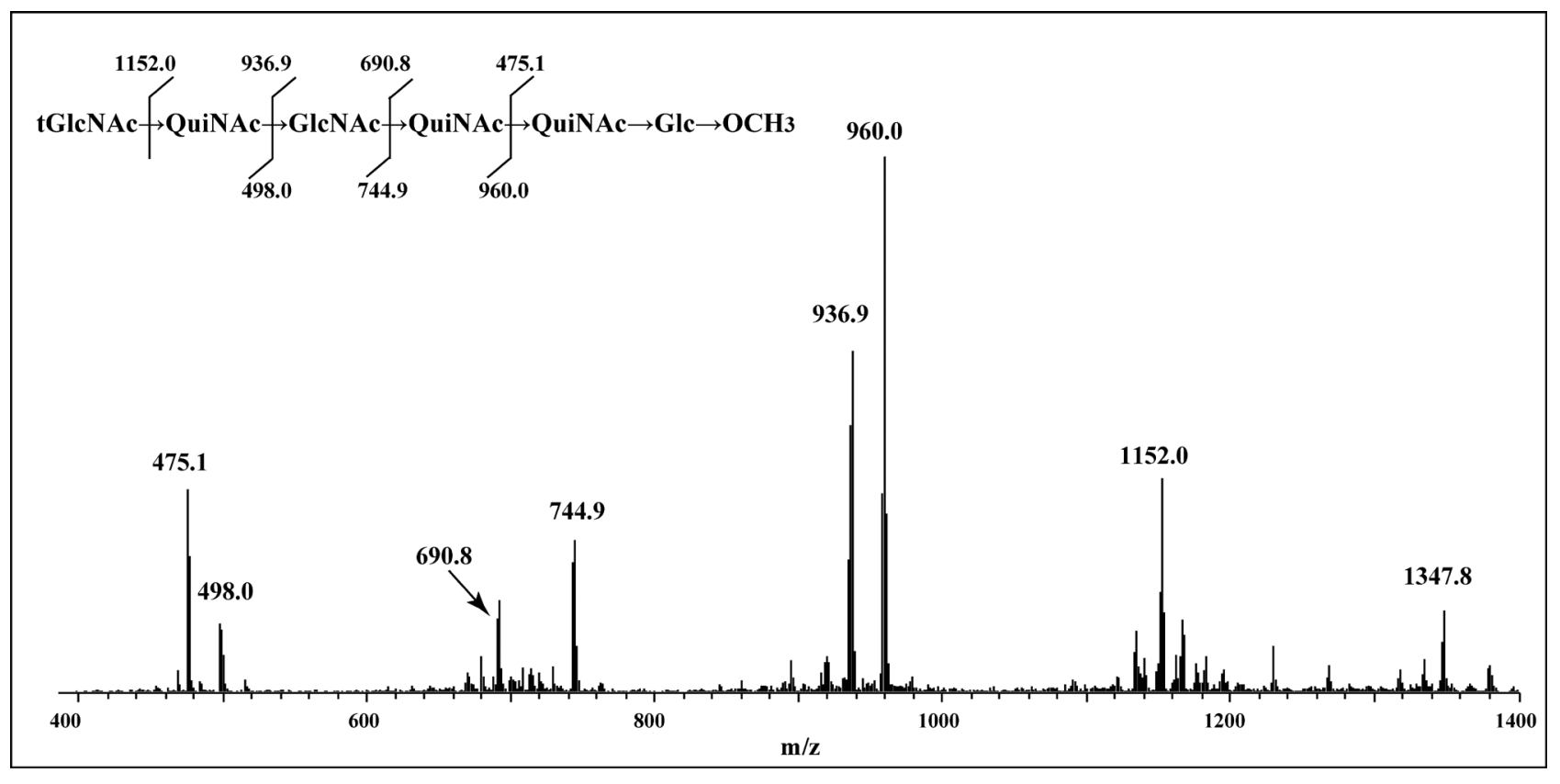

Supplement 8

ESI-MS ${ }^{2}$ spectrum and the fragmentation scheme of the glycoform Hex10 at $m / z 1412$; $t$, terminal 


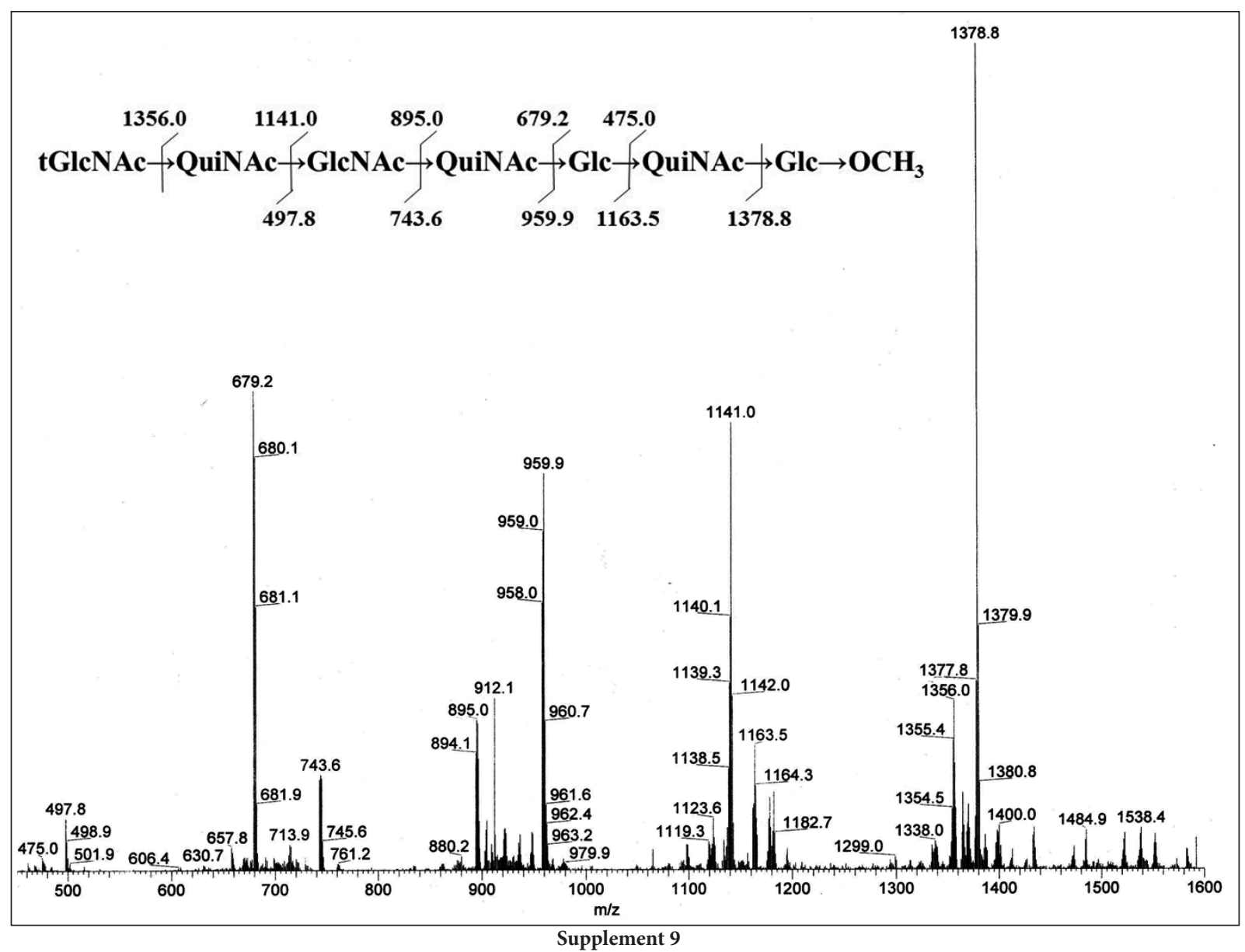

ESI-MS $^{2}$ spectrum and the fragmentation scheme of the glycoform Hex11 at $m / z$ 1616; t terminal
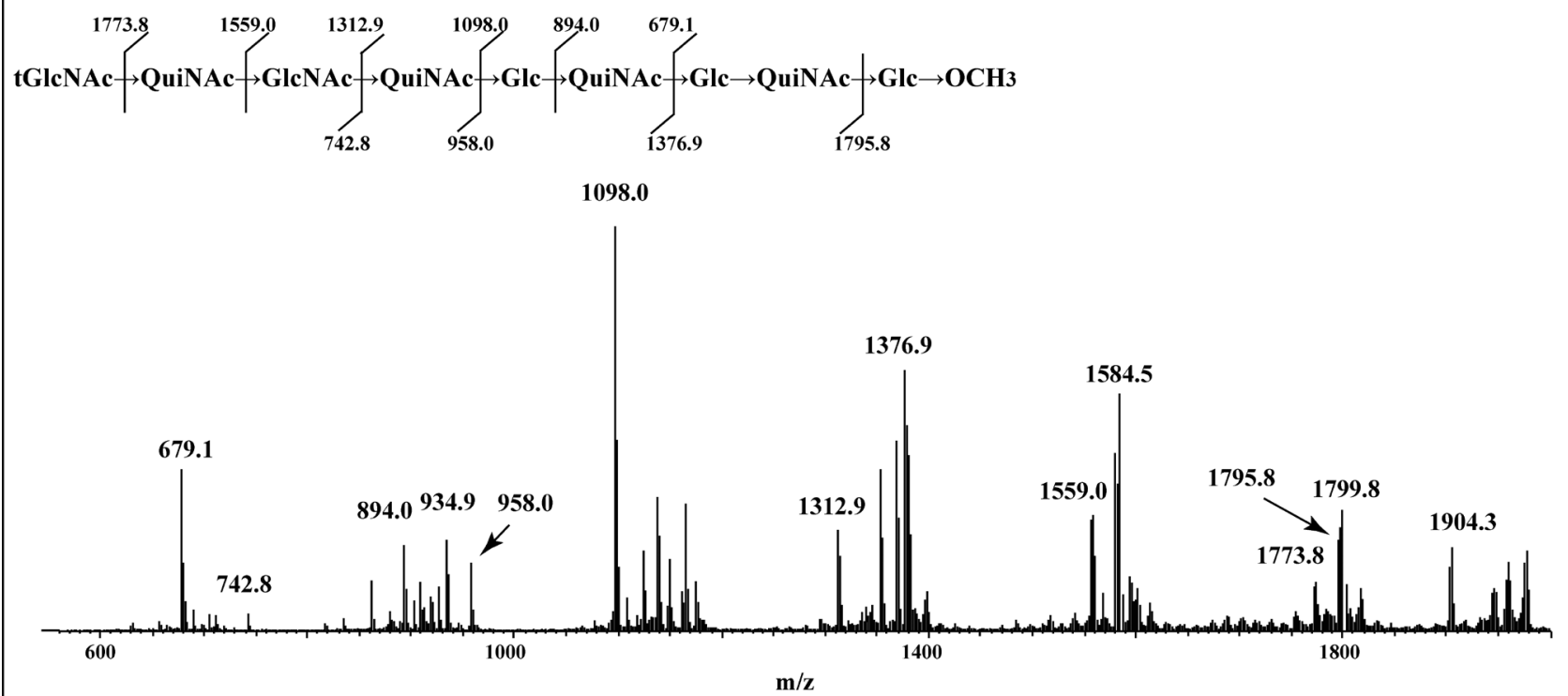

Supplement 10

ESI-MS ${ }^{2}$ spectrum and the fragmentation scheme of the glycoform Hex12 at $m / z 2035$; $t$, terminal 